\title{
ALTERAÇÕES EM ATRIBUTOS QUÍMICOS DE SOLO CULTIVADO COM CANA-DE-AÇÚCAR E ADIÇÃO DE VINHAÇA ${ }^{1}$
}

\author{
Rubens Pessoa de Barros 2 , Pedro Roberto Almeida Viégas ${ }^{3}$, Thiago Lima da Silva ${ }^{3}$, Richard Matos de Souza ${ }^{3}$, \\ Leandro Barbosa ${ }^{3}$, Ricardo Almeida Viégas ${ }^{4}$, Marcos Cabral de Vasconcellos Barretto ${ }^{3}$, Alberto Soares de Melo ${ }^{5}$
}

\begin{abstract}
CHEMICAL CHANGES IN SOILS CULTIVATED

WITH SUGARCANE AND ADDITION OF VINASSE

The research aimed to evaluate the effect of vinasse on the chemical properties of a eutrophic Alfissol cultivated with sugarcane (S. officinarum spp.), for 10 years, in two areas of the Usina São José do Pinheiro, located in the city of Laranjeiras, Sergipe State, Brazil. The experimental design was randomized blocks, with ten replications, which consisted of two areas (Area A - cultivated with sugarcane and fertirrigated with vinasse, for over 10 years; and Area B - cultivated with sugarcane without vinasse fertigation) and three soil sampling depths (treatments): 0-15 cm, 15-30 cm, and 30-45 cm. The experimental areas dimensions were $50 \mathrm{~m}$ x $20 \mathrm{~m}\left(1,000 \mathrm{~m}^{2}\right)$ and 30 samples were collected from each site. After the analysis of $\mathrm{pH}$, soil organic matter (SOM), and levels of macro and micronutrients from soil samples, it was observed that the addition of vinasse to sugarcane crops, for ten years, has altered the soil chemical properties, with increase of soil organic matter and macronutrient content and decrease of micronutrients availability. Based on the results obtained, it was concluded that the application of vinasse increases the fertility of soil cultivated with sugarcane.
\end{abstract}

KEY-WORDS: Saccharum officinarum; organic matter; soil conditioning; macronutrients; micronutrients.

\section{INTRODUÇÃO}

Os mercados, interno e externo, estão à procura de combustível limpo e renovável, seja para adição à gasolina, seja para uso em automóveis bicombustíveis. A tendência atual do setor da agroenergia brasileira é investir no avanço da cultura canavieira, em áreas agricultáveis degradadas, principalmente

\section{RESUMO}

A pesquisa teve como objetivo avaliar o efeito da aplicação da vinhaça sobre as propriedades químicas de um Argissolo Vermelho-Amarelo eutrófico, cultivado com cana-deaçúcar (S. officinarum spp.), por 10 anos, em duas áreas da Usina São José do Pinheiro, localizada no Município de Laranjeiras, Estado de Sergipe. O delineamento experimental foi em blocos casualizados, com dez repetições, sendo constituído por duas áreas (Área A - cultivada com cana-de-açúcar e fertirrigada com vinhaça, por mais de 10 anos; e Área B - cultivada com canade-açúcar sem fertirrigação com vinhaça) e três profundidades $(0-15 \mathrm{~cm}, 15-30 \mathrm{~cm}$ e $30-45 \mathrm{~cm})$ de amostragem do solo (tratamentos). As áreas experimentais apresentaram dimensões de $50 \mathrm{~m}$ x $20 \mathrm{~m}\left(1.000 \mathrm{~m}^{2}\right)$, sendo retiradas 30 amostras de cada área, por meio de tradagem. Após as análises de $\mathrm{pH}$, matéria orgânica do solo (MOS) e dos teores de macro e micronutrientes das amostras do solo, verificou-se que a adição de vinhaça à lavoura de cana-de-açúcar, por dez anos, alterou as propriedades químicas do solo, sendo observado incremento da matéria orgânica e dos teores de macronutrientes e diminuição da disponibilidade de micronutrientes. Com base nos resultados obtidos, conclui-se que a aplicação de vinhaça aumenta a fertilidade do solo destinado ao cultivo de cana-de-açúcar.

PALAVRAS-CHAVE: Saccharum officinarum; condicionamento do solo; matéria orgânica; macronutrientes; micronutrientes.

sobre áreas de pastagens extensivas (Vieira Júnior et al. 2008, Castro et al. 2010).

A cana-de-açúcar (Saccharum officinarum spp.) tem sido cultivada de forma contínua e intensa, ao longo dos séculos, nos Tabuleiros Costeiros do Nordeste. Esta região apresenta precipitação pluviométrica favorável, o que permite o desenvolvimento de um sistema de produção agrícola com

1. Trabalho recebido em jun./2009 e aceito para publicação em set./2010 (nº registro: PAT 6422/ DOI: 10.5216/pat.v40i3.6422).

2. Universidade Estadual de Alagoas, Núcleo de Ensino-Pesquisa e Aplicação em Biologia, Departamento de Biologia, Arapiraca, AL, Brasil.E-mail: pessoa.rubens@gmail.com.

3. Universidade Federal de Sergipe, Centro de Ciências Biológicas e da Saúde, Departamento de Engenharia Agronômica, São Cristóvão, SE, Brasil.E-mails: pviegas@ufs.br, thiagolim@hotmail.com, richard-matos@hotmail.com, leandro@ufs.br,mbarreto@ufs.br. 4. Universidade Federal de Campina Grande, Centro de Saúde e Tecnologia Rural, Departamento de Engenharia Florestal, Patos, PB, Brasil.E-mail: raviegas@uol.com.br.

5. Universidade Estadual da Paraíba, Centro de Ciências Humanas e Agrárias, Departamento de Ciências Agrárias e Exatas, Catolé do Rocha, PB, Brasil.E-mail: alberto@uepb.edu.br. 
baixo risco de secas periódicas (Jacomine 1974, Sant'anna 2007).

O agroecossistema da cana-de-açúcar tem sido foco de pesquisas, por possuir relativa expressão econômica e ocupação fundiária, gerando grande impacto pelos subprodutos oriundos da produção de açúcar e álcool. Com, aproximadamente, 5,57 milhões de hectares plantados e uma safra anual de cerca de 427 milhões de toneladas, o Brasil é o maior produtor mundial de cana. A safra 2006/2007 atingiu 29,8 milhões de toneladas de açúcar e 17,7 bilhões de litros de álcool. Este montante movimenta cerca de 50 bilhões de reais por ano, com faturamentos diretos e indiretos, o que corresponde a $1,5 \%$ do PIB brasileiro (Unica 2007, Brasil 2007).

$\mathrm{Na}$ região Nordeste, a cana-de-açúcar tem relevância econômica e social, há séculos, e o Estado de Sergipe é o $7^{\circ}$ no ranking nacional, em termos de produção, sendo produzidas 786,7 toneladas $a^{-1}$ de açúcar e 1.609, 8 toneladas ano ${ }^{-1}$ de álcool (Conab 2008).

Dentre os resíduos gerados pela indústria sucroalcooleira, a vinhaça é o mais importante, devido ao grande volume produzido. Para a produção de um litro de álcool, são gerados cerca de 13 litros de vinhaça, sendo que esta quantidade depende da tecnologia utilizada nas usinas ou destilarias. Este resíduo apresenta elevada concentração de nutrientes, principalmente potássio $(\mathrm{K})$, e de matéria orgânica, apresentando, portanto, alto potencial poluidor.

A alternativa mais viável para descarte do resíduo é a aplicação da vinhaça aos solos cultivados com cana-de-açúcar (Freire \& Cortez 2000). Dentre os efeitos associados à adição de vinhaça ao solo estão a elevação do pH, que, segundo Rodella et al. (1983), é transiente, podendo retornar aos valores originais, após determinado período de tempo, e a elevação da CTC, devido ao grande aporte de matéria orgânica, que diminui o risco de lixiviação de cátions e o aumento dos teores de macronutrientes no solo (Glória \& Orlando Filho 1983). Portanto, esta prática tem sido associada à melhoria da fertilidade do solo, devido ao fornecimento de nutrientes e incremento de matéria orgânica propiciado pelo resíduo. O uso agrícola da vinhaça e, consequentemente, a redução dos riscos ambientais, fez com que o resíduo adquirisse valor econômico, a partir de 1975, passando de agente poluidor a condicionador químico e físico do solo (Rossetto 1987).
Atualmente, a prática de aplicação da vinhaça na lavoura, por meio da fertirrigação, é adotada na maioria das usinas, com tecnologia bem conhecida, existindo inúmeros ensaios que comprovam os resultados positivos obtidos na produtividade agrícola, associados à economia dos adubos minerais (Penatti et al. 1988). No entanto, é importante destacar que poucas pesquisas foram desenvolvidas na região Nordeste. Neste contexto, o objetivo do trabalho foi avaliar o efeito da aplicação da vinhaça sobre as propriedades químicas de solos cultivados com canade-açúcar, por um período de 10 anos.

\section{MATERIAL E MÉTODOS}

O experimento foi realizado em duas áreas da Usina São José do Pinheiro, localizada no município de Laranjeiras, SE (1048'29''S e 37 09'37'WO), em solo classificado como Argissolo Vermelho-Amarelo eutrófico. De acordo com a classificação de Köppen, a região possui clima tipo As (tropical-chuvoso, com verão seco) e pluviometria em torno de $1.200 \mathrm{~mm}$ anuais, com chuvas concentradas nos meses de abril a setembro. O relevo da área é plano a suave ondulado, com predominância de platôs característicos dos Tabuleiros Costeiros.

$\mathrm{O}$ solo, de ambas as áreas, foi adubado com $60 \mathrm{~kg} \mathrm{ha}^{-1}$ de N, $100 \mathrm{~kg} \mathrm{ha}^{-1}$ de $\mathrm{P}_{2} \mathrm{O}_{5}$ e $90 \mathrm{~kg} \mathrm{ha}^{-1}$ de $\mathrm{K}_{2} \mathrm{O}$, empregando-se ureia e misturas N-P-K comerciais disponíveis na usina, conforme recomendações baseadas na análise do solo, à exceção da área $\mathrm{A}$, que não recebeu as doses de $\mathrm{K}$, cuja fonte foi a vinhaça $\left(150 \mathrm{~m}^{3} \mathrm{ha}^{-1}\right)$. A composição química da vinhaça utilizada está descrita na Tabela 1.

O delineamento experimental foi em blocos casualizados, em esquema fatorial, sendo constituído

Tabela 1. Caracterização físico-química da vinhaça in natura, utilizada na fertirrigação da área A.

\begin{tabular}{lr}
\hline \multicolumn{1}{c}{ Variáveis } & \multicolumn{1}{c}{ Valores } \\
\hline $\mathrm{pH}$ em água $(1: 2,5)$ & 4,56 \\
$\mathrm{DBO} *\left(\mathrm{mg} \mathrm{L}^{-1}\right)$ & $21.275,00$ \\
$\mathrm{DQO} *\left(\mathrm{mg} \mathrm{L}^{-1}\right)$ & $48.860,00$ \\
$\mathrm{CE}^{*}\left(\mathrm{dS} \mathrm{m}^{-}\right)$ & 9,65 \\
$\mathrm{SDT}\left(\mathrm{mg} \mathrm{L}^{-1}\right)$ & 19,00 \\
$\mathrm{~K}\left(\mathrm{mg} \mathrm{L}^{-1}\right)$ & $1.392,00$ \\
$\mathrm{P}$ total $\left(\mathrm{mg} \mathrm{L}^{-1}\right)$ & 175,00 \\
$\mathrm{Ca}\left(\left(\mathrm{mg} \mathrm{L}^{-1}\right)\right.$ & 728,00 \\
$\mathrm{Na}\left(\mathrm{mg} \mathrm{L}^{-1}\right)$ & 110,00 \\
$\mathrm{Mg}\left(\mathrm{mg} \mathrm{L}^{-1}\right)$ & 29,00 \\
\hline
\end{tabular}

* DQO - demanda química de oxigênio; DBO - demanda bioquímica de oxigênio; CE - condutividade elétrica; SDT - sólidos dissolvidos totais. 
por duas áreas cultivadas com cana-de-açúcar (Área A - fertirrigada com vinhaça; e Área B - sem fertirrigação com vinhaça), por 10 anos, e três profundidades (0-15 cm, 15-30 cm e 30-45 cm) de amostragem do solo (tratamentos), com dez repetições. Para a retirada das amostras deformadas do solo, nas três profundidades, em ambas as áreas, foi, inicialmente, marcada uma área experimental, dentro dos talhões de cana-de-açúcar, com as dimensões $50 \mathrm{~m} \times 20 \mathrm{~m}\left(1.000 \mathrm{~m}^{2}\right)$, utilizandose trado tipo holandês, para a obtenção das amostras.

As amostras de solo foram acondicionadas em sacos plásticos, lacrados, etiquetados e armazenados à temperatura ambiente, no Laboratório de Remediação de Solos do Departamento de Engenharia Agronômica da Universidade Federal de Sergipe (DEA/ UFS), até o início das análises químicas, efetuadas no Laboratório de Fertilidade do Solo do Instituto Tecnológico de Pesquisa do Estado de Sergipe (ITPS). A metodologia utilizada para as análises químicas das amostras de solo foi a da Embrapa (1997), sendo determinados, em ambas as áreas, o $\mathrm{pH}$, matéria orgânica do solo (MOS), macro e micronutrientes minerais, sódio, capacidade de troca cationnica (CTC), soma de bases (SB) e saturação por bases $(\% \mathrm{~V})$, sendo os dados obtidos submetidos a análise multivariada (MANOVA) e as médias comparadas pelo teste Tukey, a 5\% de probabilidade.

\section{RESULTADOS E DISCUSSÃO}

A adição ao solo de vinhaça, por dez anos, na área agrícola $\mathrm{A}$, proporcionou elevação do conteúdo de matéria orgânica, $\mathrm{Ca}+\mathrm{Mg}$ trocáveis, CTC, $\mathrm{V}(\%), \mathrm{SB}$, macro e micronutrientes, não havendo efeito sobre o $\mathrm{pH}$, quando comparada com a área $\mathrm{B}$ (Tabelas 2 e 3). Resultados semelhantes foram obtidos por Canellas et al. (2003), os quais estudaram a aplicação de vinhaça, durante 35 anos, em cana-deaçúcar queimada e crua.
De acordo com Rodella et al. (1983), os efeitos de elevação do pH do solo podem ser efêmeros, podendo retornar aos valores originais, após determinado período de tempo. De fato, no presente trabalho, não houve efeito da vinhaça sobre o $\mathrm{pH}$ do solo. Segundo Silva \& Ribeiro (1998), o pH dos solos tratados com vinhaça aumenta, principalmente em áreas cultivadas há mais tempo. Todavia, nos primeiros dez dias após a aplicação, o pH pode sofrer redução considerável, para, posteriormente, elevar-se abruptamente, podendo alcançar valores superiores a 7,0, provavelmente devido à ação de micro-organismos.

A elevação da CTC pode ser justificada pelo grande aporte de matéria orgânica representado pelas adições da vinhaça. Pela característica coloidal da matéria orgânica contida na vinhaça, sua adição confere ao solo maior quantidade de cargas negativas, diminuindo o potencial de lixiviação de cátions (Glória \& Orlando Filho 1983).

O efeito da aplicação da vinhaça, para complementar as adubações de manutenção da cana-deaçúcar, pode ser observado no teor de K, no perfil do solo da área A, o qual foi maior, em relação ao teor observado na área B. Em experimento em área fertirrigada com vinhaça, Gonzalo et al. (2005) ob-

Tabela 2. Matéria orgânica do solo (MOS), pH, capacidade de troca catiônica (CTC pH7), saturação por bases $(\% \mathrm{~V})$ e soma de bases (SB) de solo cultivado com canade-açúcar fertirrigada (Área A) e sem fertirrigação com vinhaça (Área B), por 10 anos (São Cristóvão, SE, 2008).

\begin{tabular}{lccccc}
\hline \multirow{2}{*}{ Local } & $\mathrm{MOS}$ & $\mathrm{pH}$ & $\mathrm{CTC}(\mathrm{pH} 7)$ & $\mathrm{V}$ & $\mathrm{SB}$ \\
\cline { 2 - 6 } & $\mathrm{g} \mathrm{dm}^{-3}$ & $\mathrm{H}_{2} \mathrm{O}$ & $\mathrm{cmol}_{\mathrm{c}} \mathrm{dm}^{-3}$ & $\%$ & $\mathrm{cmolc} \mathrm{dm}^{-3}$ \\
\hline Área A & $33,9 \mathrm{a}^{*}$ & $7,0 \mathrm{a}$ & $42,7 \mathrm{a}$ & $98,2 \mathrm{a}$ & $41,5 \mathrm{a}$ \\
Área B & $20,7 \mathrm{~b}$ & $6,2 \mathrm{a}$ & $12,5 \mathrm{~b}$ & $84,3 \mathrm{~b}$ & $10,5 \mathrm{~b}$ \\
\hline CV (\%) & 19,70 & 7,15 & 19,99 & 20,25 & 20,10 \\
\hline DMS (5\%) & 2,80 & 0,30 & 2,66 & 2,55 & 2,70 \\
\hline * Médias seguidas de mesma letra minúscula, na coluna, não diferem entre si, a \\
5\% de probabilidade, pelo teste Tukey.
\end{tabular}

Tabela 3. Comparação dos teores de $\mathrm{Ca}+\mathrm{Mg}, \mathrm{K}, \mathrm{P}, \mathrm{Na}, \mathrm{Fe}, \mathrm{Cu}, \mathrm{Mn}$ e Zn de um solo cultivado com cana-de-açúcar, fertirrigado com vinhaça (Área A) e não fertirrigado (Área B), por 10 anos (São Cristóvão, SE, 2008).

\begin{tabular}{|c|c|c|c|c|c|c|c|c|}
\hline \multirow{2}{*}{ Local } & $\mathrm{Ca}+\mathrm{Mg}$ & $\mathrm{K}$ & $\mathrm{P}$ & $\mathrm{Na}$ & $\mathrm{Fe}$ & $\mathrm{Cu}$ & $\mathrm{Mn}$ & $\mathrm{Zn}$ \\
\hline & $\mathrm{cmol}_{\mathrm{c}} \mathrm{dm}^{-3}$ & \multicolumn{2}{|c|}{$\mathrm{mg} \mathrm{dm}^{-3}$} & $\mathrm{cmol}_{\mathrm{c}} \mathrm{dm}^{-3}$ & \multicolumn{4}{|c|}{$\mathrm{mg} \mathrm{dm}^{-3}$} \\
\hline Área A & $40,6 a^{*}$ & $271,8 \mathrm{a}$ & $19,3 \mathrm{a}$ & $0,23 \mathrm{a}$ & $98,17 \mathrm{a}$ & $166,59 \mathrm{~b}$ & $0,051 \mathrm{~b}$ & $97,80 \mathrm{~b}$ \\
\hline Área B & $10,0 \mathrm{~b}$ & $81,7 \mathrm{~b}$ & $7,7 \mathrm{~b}$ & $0,10 \mathrm{~b}$ & $84,34 \mathrm{~b}$ & $1.568,10 \mathrm{a}$ & $3,350 \mathrm{a}$ & $652,68 \mathrm{a}$ \\
\hline $\mathrm{CV}(\%)$ & 20,40 & 52,27 & 48,21 & 20,87 & 5,31 & 10,11 & 66,26 & 48,21 \\
\hline DMS (5\%) & 2,68 & 47,79 & 8,75 & 4,55 & 4,69 & 0,59 & 97,25 & 35,52 \\
\hline
\end{tabular}

* Médias seguidas de mesma letra minúscula, na coluna, não diferem entre si, a 5\% de probabilidade, pelo teste Tukey. 
servaram que o excesso de $\mathrm{K}$, na camada superficial, foi onze vezes superior à recomendação de $\mathrm{K}_{2} \mathrm{O}$ por hectare, para cana-soca. Como o K tem alto potencial de lixiviação, é importante considerar a necessidade de $\mathrm{K}$ da cultura e a capacidade sortiva do solo, na tomada de decisão da dose de vinhaça a ser aplicada, a fim de minimizar os impactos ambientais ocasionados pelo uso agrícola do resíduo.

Dessa forma, o estudo do uso de vinhaça, em solos de área canavieira, torna-se bastante importante para recomendação agronômica do resíduo. Cambuim (1983), ao avaliar o efeito da aplicação de três doses de vinhaça $\left(0 \mathrm{~m}^{3} \mathrm{ha}^{-1}, 200 \mathrm{~m}^{3} \mathrm{ha}^{-1} \mathrm{e}\right.$ $\left.400 \mathrm{~m}^{3} \mathrm{ha}^{-1}\right)$, em diferentes tempos de incubação, verificou acréscimo nos teores de $\mathrm{K}$ e redução de $\mathrm{Na}$ trocável, observando, também, que, via de regra, o período de incubação atuou mais diretamente sobre estes efeitos que as doses testadas. Camargo et al. (1983) avaliaram a saturação de K e a capacidade de troca de cátions, em amostras de Latossolo textura média, as quais foram tratadas, por longo tempo, com diferentes quantidades de vinhaça, intercaladas com períodos de repouso do solo. Os autores observaram correlação positiva das doses de vinhaça com a saturação por K e CTC do solo. No entanto, ressaltaram que o aumento excessivo da concentração de K no solo, em algumas áreas, pode ocasionar desequilíbrios nutricionais, dependendo da cultura a ser implantada.

A comparação dos atributos químicos entre as áreas A e B, nas três profundidades (Tabela 4), indica que houve diferença significativa da aplicação de vinhaça entre as áreas, confirmando a importância deste subproduto na melhoria dos atributos do solo. Orlando Filho et al. (1983) estudaram a aplicação de vinhaça e a elevação do $\mathrm{pH}$, por um período de 20 anos, e verificaram que não houve efeito negativo nas propriedades químicas dos solos estudados. A aplicação deste subproduto elevou os teores de $\mathrm{K}, \mathrm{Ca}, \mathrm{Mg}$, a soma de bases e a CTC do solo.

Entre as três profundidades avaliadas, em cada área (Tabela 4), observou-se diferença significativa nos atributos químicos, especialmente para o solo da área $\mathrm{A}$ (fertirrigada com vinhaça), que apresentou os maiores valores, principalmente na profundidade 0-15 cm. O teor de MOS diferiu, significativamente, entre as três profundidades, verificando-se, para ambas as áreas, maior valor na camada $0-15 \mathrm{~cm}$ (Tabela 4). Este aumento do teor da MOS pode contribuir com a maior retenção de cátions e diminuir suas perdas por lixiviação.

Para os teores de macro e micronutrientes, houve diferença significativa $(\mathrm{p}<0,05)$ (Tabela 5). Nas áreas avaliadas, verificou-se um aporte maior de macronutrientes, principalmente nas camadas 0-15 cm e $15-30 \mathrm{~cm}$ (Tabela 5). Entre as três profundidades, o $\mathrm{K}$ apresentou os maiores teores na camada $0-15 \mathrm{~cm}$, o que representou aumento de $2,63 \%$, quando comparado com a profundidade $30-45 \mathrm{~cm}$. Pode-se inferir que, apesar da sua forma líquida, a vinhaça tende a concentrar o $\mathrm{K}$ nas camadas superiores do solo da área fertirrigada. Tal comportamento foi observado na superfície do solo da área fertirrigada, bem como a diminuição dos teores em profundidade, fato verificado por vários pesquisadores, em outras culturas (Centurion et al. 1985, Eltz et al. 1989, Bayer \& Mielniczuk 1997,

Tabela 4. Matéria orgânica do solo (MOS), pH, capacidade de troca catiônica (CTC pH7), saturação por bases (V\%) e soma de bases (SB), de solo cultivado com cana-de-açúcar, fertirrigado com vinhaça (Área A) e sem fertirrigação (Área B), por 10 anos, em três profundidades (São Cristóvão, SE, 2008).

\begin{tabular}{|c|c|c|c|c|c|c|}
\hline \multirow{2}{*}{ Parâmetros } & \multirow{2}{*}{ Áreas } & \multicolumn{3}{|c|}{ Profundidades $(\mathrm{cm})$} & \multirow{2}{*}{ DMS } & \multirow{2}{*}{$\frac{C V}{(\%)}$} \\
\hline & & $0-15$ & $15-30$ & $30-45$ & & \\
\hline \multirow{2}{*}{$\operatorname{MOS}\left(\mathrm{g} \mathrm{dm}^{-3}\right)$} & $\mathrm{A}$ & $41,7 \mathrm{~A} \mathrm{a}^{*}$ & $32,7 \mathrm{~A} \mathrm{~b}$ & $27,5 \mathrm{Ac}$ & \multirow{2}{*}{4,13} & \multirow{2}{*}{19,80} \\
\hline & B & $25,3 \mathrm{~B} \mathrm{a}$ & $19,9 \mathrm{~B} \mathrm{~b}$ & $16,9 \mathrm{~B} \mathrm{c}$ & & \\
\hline \multirow{2}{*}{$\mathrm{pH}$} & $\mathrm{A}$ & $7,0 \mathrm{~A} \mathrm{a}$ & $7,1 \mathrm{Ab}$ & $7,1 \mathrm{Ab}$ & \multirow{2}{*}{0,45} & \multirow{2}{*}{8,79} \\
\hline & $\mathrm{B}$ & $6,4 \mathrm{~B} \mathrm{a}$ & $6,3 \mathrm{~B} \mathrm{a}$ & $5,9 \mathrm{~B} \mathrm{~b}$ & & \\
\hline \multirow{2}{*}{ CTC pH7 $\left(\mathrm{cmol}_{\mathrm{c}} \mathrm{dm}^{-3}\right)$} & A & $41,7 \mathrm{~A} \mathrm{a}$ & $45,8 \mathrm{~A} \mathrm{~b}$ & $40,6 \mathrm{~A} \mathrm{c}$ & \multirow[b]{2}{*}{3,92} & \multirow{2}{*}{18,82} \\
\hline & $\mathrm{B}$ & $12,0 \mathrm{~B} \mathrm{a}$ & $12,7 \mathrm{~B} \mathrm{~b}$ & $12,8 \mathrm{~B} \mathrm{c}$ & & \\
\hline \multirow[b]{2}{*}{ V\% } & $\mathrm{A}$ & $98,1 \mathrm{~A} \mathrm{~b}$ & $98,6 \mathrm{~A} \mathrm{~b}$ & $98,3 \mathrm{~A} \mathrm{a}$ & \multirow{2}{*}{3,76} & \multirow{2}{*}{5,41} \\
\hline & B & $87,0 \mathrm{~B}$ a & $85,7 \mathrm{~B} \mathrm{~b}$ & $80,2 \mathrm{~B} \mathrm{c}$ & & \\
\hline \multirow{2}{*}{$\mathrm{SB}\left(\mathrm{cmol}_{\mathrm{c}} \mathrm{dm}^{-3}\right)$} & A & $40,9 \mathrm{~A} \mathrm{a}$ & $45,1 \mathrm{~A} \mathrm{~b}$ & $39,9 \mathrm{~A} \mathrm{a}$ & \multirow{2}{*}{3,98} & \multirow{2}{*}{20,10} \\
\hline & B & 10,4 B b & $10,9 \mathrm{~B} \mathrm{~b}$ & $10,3 \mathrm{~B} \mathrm{~b}$ & & \\
\hline
\end{tabular}

* Médias seguidas de mesma letra maiúscula, na coluna, e minúscula, na linha, não diferem entre si, a 5\% de probabilidade, pelo teste Tukey. 
Tabela 5. Valores médios dos teores de $\mathrm{Ca}+\mathrm{Mg}, \mathrm{Na}, \mathrm{K}, \mathrm{P}, \mathrm{Fe}, \mathrm{Cu}, \mathrm{Mn}$ e Zn, em solo cultivado com cana-de-açúcar, fertirrigado com vinhaça (Área A) e sem fertirrigação (Área B), por 10 anos, em três profundidades (São Cristóvão, SE, 2008).

\begin{tabular}{|c|c|c|c|c|c|c|}
\hline \multirow{2}{*}{ Parâmetros } & \multirow{2}{*}{ Áreas } & \multicolumn{3}{|c|}{ Profundidades $(\mathrm{cm})$} & \multirow{2}{*}{ DMS } & \multirow{2}{*}{$\begin{array}{l}\mathrm{CV} \\
(\%)\end{array}$} \\
\hline & & $0-15$ & $15-30$ & $30-45$ & & \\
\hline \multirow{2}{*}{$\mathrm{Ca}+\mathrm{Mg}\left(\mathrm{cmolc} \mathrm{dm}^{-3}\right)$} & $\mathrm{A}$ & $38,79 \mathrm{~A} \mathrm{a}^{*}$ & 43,86 A a & $38,94 \mathrm{Ab}$ & \multirow{2}{*}{3,95} & \multirow{2}{*}{20,40} \\
\hline & $\mathrm{B}$ & $10,07 \mathrm{~B} \mathrm{a}$ & $10,60 \mathrm{~B} \mathrm{a}$ & $9,96 \mathrm{~B} \mathrm{a}$ & & \\
\hline \multirow{2}{*}{$\mathrm{K}\left(\mathrm{mg} \mathrm{dm}^{-3}\right)$} & A & $425,80 \mathrm{~A} \mathrm{a}$ & $227,83 \mathrm{Ab}$ & $161,87 \mathrm{Ac}$ & \multirow{2}{*}{70,37} & \multirow{2}{*}{52,27} \\
\hline & $\mathrm{B}$ & $97,62 \mathrm{~B} \mathrm{a}$ & $75,49 \mathrm{~B} \mathrm{~b}$ & $72,02 \mathrm{~B} \mathrm{~b}$ & & \\
\hline \multirow{2}{*}{$\mathrm{P}\left(\mathrm{mg} \mathrm{dm}^{-3}\right)$} & $\mathrm{A}$ & $28,74 \mathrm{~A} \mathrm{a}$ & $16,92 \mathrm{Ab}$ & $12,36 \mathrm{Ac}$ & \multirow{2}{*}{13,25} & \multirow{2}{*}{48,21} \\
\hline & $\mathrm{B}$ & $8,83 \mathrm{~B} \mathrm{a}$ & $7,04 \mathrm{~B} \mathrm{a}$ & $7,32 \quad \mathrm{~B} \mathrm{a}$ & & \\
\hline \multirow{2}{*}{$\mathrm{Na}\left(\mathrm{mg} \mathrm{dm}^{-3}\right)$} & A & $54,00 \mathrm{~A} \mathrm{a}$ & 55,89 A a & $49,02 \mathrm{~A} \mathrm{a}$ & \multirow{2}{*}{6,70} & \multirow{2}{*}{20,87} \\
\hline & $\mathrm{B}$ & $19,49 \mathrm{~B} \mathrm{a}$ & $18,93 \mathrm{~B} \mathrm{a}$ & $30,50 \mathrm{~B} \mathrm{~b}$ & & \\
\hline \multirow{2}{*}{$\mathrm{Fe}\left(\mathrm{mg} \mathrm{dm}^{-3}\right)$} & A & $98,10 \mathrm{~A} \mathrm{~b}$ & 98,73 A a & 97,67 A c & \multirow{2}{*}{6,89} & \multirow{2}{*}{5,31} \\
\hline & $\mathrm{B}$ & $87,10 \mathrm{~B} \mathrm{~b}$ & $85,61 \mathrm{~B} \mathrm{~b}$ & $80,30 \quad \mathrm{~B} \mathrm{~b}$ & & \\
\hline \multirow{2}{*}{$\mathrm{Cu}\left(\mathrm{mg} \mathrm{dm}^{-3}\right)$} & $\mathrm{A}$ & $173,45 \mathrm{~A} \mathrm{~b}$ & $142,42 \mathrm{Ab}$ & $183,91 \mathrm{Ab}$ & \multirow{2}{*}{0,87} & \multirow{2}{*}{10,11} \\
\hline & $\mathrm{B}$ & $2.301,20 \mathrm{~B} \mathrm{~b}$ & $967,60 \mathrm{~B} \mathrm{~b}$ & $1.435,50 \mathrm{~B} \mathrm{~b}$ & & \\
\hline \multirow{2}{*}{$\operatorname{Mn}\left(\mathrm{mg} \mathrm{dm}^{-3}\right)$} & A & $0,070 \mathrm{~A} \mathrm{~b}$ & $0,013 \mathrm{~A} \mathrm{~b}$ & $0,070 \mathrm{~A} \mathrm{a}$ & \multirow{2}{*}{143,20} & \multirow{2}{*}{66,26} \\
\hline & $\mathrm{B}$ & $4,147 \mathrm{~B} \mathrm{~b}$ & $2,696 \mathrm{~B} \mathrm{~b}$ & $3,221 \mathrm{~B} \mathrm{a}$ & & \\
\hline \multirow{2}{*}{$\mathrm{Zn}\left(\mathrm{mg} \mathrm{dm}{ }^{-3}\right)$} & $\mathrm{A}$ & $105,61 \mathrm{Ab}$ & $86,04 \mathrm{Ab}$ & $101,74 \mathrm{Ab}$ & \multirow{2}{*}{52,30} & \multirow{2}{*}{48,21} \\
\hline & $\mathrm{B}$ & $826,20 \mathrm{~B} \mathrm{~b}$ & $531,63 \quad \mathrm{~B} \mathrm{~b}$ & $600,20 \mathrm{~B} \mathrm{~b}$ & & \\
\hline
\end{tabular}

* Médias seguidas de mesma letra maiúscula, na coluna, e minúscula, na linha, não diferem entre si, a 5\% de probabilidade, pelo teste Tukey.

Souza \& Alves 2003). Cunha et al. (1981), ao avaliarem a vinhaça como fertilizante e condicionador de solos, verificaram que a acumulação de $\mathrm{K}$ no perfil não foi grande, ficando este elemento retido na camada de $0,50 \mathrm{~m}$ de profundidade, sendo que sua lixiviação foi pequena, acompanhando a drenagem interna no perfil.

Houve acréscimo significativo de $\mathrm{P}$, nas três profundidades do solo da área $\mathrm{A}$, quando comparada com a área B. Este aporte evidencia a importância da vinhaça como fornecedora deste nutriente para a cultura da cana-de-açúcar. Busato et al. (2005), estudando a aplicação de vinhaça em diferentes profundidades (0,0-0,20 m e 0,20-0,40 m), verificaram que, na área com aplicação de vinhaça, foram observadas diferenças de $210 \%$ e $267 \%$, respectivamente, no compartimento P-disponível, em relação à área que não recebeu este subproduto.

Observou-se variação nos teores de micronutrientes, entre as áreas e as profundidades (Tabela 5). Nas camadas 0-15 cm e 15-30 cm da área $B$, a qual não recebeu vinhaça, houve maior concentração de $\mathrm{Cu}$, $\mathrm{Mn}$ e $\mathrm{Zn}$. Provavelmente, a menor concentração dos referidos micronutrientes no perfil do solo da área $\mathrm{A}$ pode ter ocorrido pela elevação do $\mathrm{pH}$ do solo, que diminuiu a solubilização desses íons. Carneiro et al. (2004) verificaram o mesmo comportamento, quando adicionaram vinhaça ao solo. Segundo Freire \& Cortez (2000), o aumento de $\mathrm{pH}$ proporcionado pela vinhaça pode ser atribuído às reações de redução que consomem íons $\mathrm{H}+$, presentes no solo, resultando no abaixamento do potencial redox.

Para o Fe, houve diferença significativa entre as três profundidades somente na área $\mathrm{A}$.

\section{CONCLUSÕES}

1. A adição de vinhaça à lavoura de cana-de-açúcar, por dez anos, alterou, positivamente, as propriedades químicas do solo, proporcionando melhoria na fertilidade, pelo maior aporte de macronutrientes no perfil.

2. A aplicação de vinhaça reduziu a disponibilidade de micronutrientes.

\section{REFERÊNCIAS}

BAYER, C.; MIELNICZUK, J. Nitrogênio total de um solo submetido a diferentes métodos de preparo e sistemas de cultura. Revista Brasileira de Ciência do Solo, Viçosa, v. 21, n. 2, p. 235-239, 1997.

BRASIL. Ministério da Agricultura. Balanço nacional da cana-de-açúcar e agroenergia. 2007. Disponível em: $<\mathrm{http}$ ://www.agricultura.gov.br>. Acesso em: 13 maio 2008.

BUSATO, J. G.; CANELLAS, L. P.; VELLOSO, A. C. X. Fósforo num Cambissolo cultivado com cana-de-açúcar por longo tempo: I - Fracionamento sequencial. Revista Brasileira de Ciência do Solo, Viçosa, v. 29, n. 6, p. 935944, 2005. 
CAMARGo, O. A.; VAlAdARES, J. M. A. S.; GERALDI, R. N. Características químicas e fisicas de solo que recebeu vinhaça por longo tempo. Campinas: Instituto Agronômico de Campinas, 1983. (Boletim científico, 76).

CAMBUIM, F. A. A ação da vinhaça sobre a retenção de umidade, $\mathrm{pH}$, acidez total, acumulação e lixiviação de nutrientes em solo arenoso. 1983. 133 f. Dissertação (Mestrado em Ciência do Solo)-Universidade Federal Rural de Pernambuco, Recife, 1983.

CANELlas, L. P. et al. Propriedades químicas de um cambissolo cultivado com cana-de-açúcar, com preservação do palhiço e adição de vinhaça por longo tempo. Revista Brasileira de Ciência do Solo, Viçosa, v. 27, n. 5, p. 935-944, 2003.

CARNEIRO, C. E. A. et al. Alterações químicas no solo induzidas pela aplicação superficial de palha de cana-deaçúcar, calcário e vinhaça. Semina, Londrina, v. 25, n. 4 , p. 265-272, 2004.

CASTRO, S. S. de et al. A expansão da cana-de-açúcar no Cerrado e no Estado de Goiás: elementos para uma análise espacial do processo. Boletim Goiano de Geografia, Goiânia, v. 30, n. 1, p. 171-190, 2010.

CENTURION, J. F.; DEMATTÊ, J. L. I.; FERNANDES, F. M. Efeitos de sistemas de preparo nas propriedades químicas de um solo sob Cerrado cultivado com soja. Revista Brasileira de Ciência do Solo, Viçosa, v. 9, n. 1, p. 267-270, 1985.

COMPANHIA NACIONAL DE ABASTECIMENTO (Conab). Acompanhamento da safra brasileira: canade-açúcar safra 2008. 2008. Disponível em <http://www. conab.gov.br/conabweb/download/safra/cana>. Acesso em: 23 nov. 2008.

CUNHA, R. C. A. et al. Effects of irrigation with vinasse and dynamics of its constituents in the soil: I - Physical and chemical aspects. Water Science and Technology, London, v. 19 , n. 8 , p. $155-165,1981$.

ELTZ, F. L. F. et al. Efeito de sistemas de preparo do solo nas propriedades físicas e químicas de um Latossolo bruno álico. Revista Brasileira de Ciência do Solo, Viçosa, v. 13, n. 2, p. 259-267, 1989.

EMPRESA BRASILEIRA DE PESQUISA AGROPECUÁRIA (Embrapa). Centro Nacional de Pesquisa de Solos. Manual de métodos de análise de solos. 2. ed. Rio de Janeiro: Embrapa, 1997.

FREIRE, W. J.; CORTEZ, L. A. B. Vinhaça de cana-deaçúcar. Guaíba: Agropecuária, 2000.

GLÓRIA, N. A.; ORLANDO FILHO, J. Aplicação de vinhaça como fertilizante. São Paulo: Coopersucar, 1983.
GONZALO, D. D. P.; CASA GRANDE, J. C.; SOARES, M. R. Lixiviação de potássio em solos adubados com vinhaça. In: CONGRESSO BRASILEIRO DE CIÊNCIA DO SOLO, 30., 2005, Recife. Anais... Recife: SBCS, 2005. 1 CD-ROM.

JACOMINE, P. K. T. Fragipans em solos de "tabuleiros": caracterização, gênese e implicações no uso agrícola. 1974. 83 f. Tese (Livre Docência em Agronomia)-Universidade Federal de Pernambuco, Recife, 1974.

ORLANDO FILHO, J.; ZAMBELLO JR., E.; AGUJARU, R. Efeito da aplicação prolongada da vinhaça nas propriedades químicas dos solos com cana-de-açúcar: estudo exploratório. STAB, Piracicaba, v. 1, n. 1, p. 2833, 1983.

PENATTI, C. P. et al. Efeitos da aplicação de vinhaça e nitrogênio na soqueira da cana-de-açúcar. Boletim Técnico Coopersucar, São Paulo, v. 44, n. 1, p. 32-38, 1988.

RODELLA, A. A.; ZAMBELLO JR., E.; ORLANDO FILHO, J. O. Calibração de análises de fósforo e potássio no solo em cana-de-açúcar: $2^{\mathrm{a}}$ aproximação. Saccharum, São Paulo, v. 28, n. 1, p. 39-42, 1983.

ROSSETTO, A. J. Utilização agronômica dos subprodutos e resíduos da indústria açucareira e alcooleira. In: PARANHOS, S. B. (Coord.). Cana-de-açúcar: cultivo e utilização. Campinas: Fundação Cargill, 1987. p. 433504.

SANT'ANNA, S. A. C. Avaliação de indicadores de qualidade do solo em áreas de cana-de-açúcar dos Tabuleiros Costeiros de Alagoas. 2007. 57 f. Dissertação (Mestrado em Agroecossistemas)-Núcleo de Estudos de Pós-Graduação em Recursos Naturais, Universidade Federal de Sergipe, São Cristóvão, 2007.

SILVA, A. J. N.; RIBEIRO, M. R. Caracterização de um Latossolo Amarelo sob cultivo contínuo de cana-de-açúcar no Estado de Alagoas: propriedades químicas. Revista Brasileira de Ciência do Solo, Viçosa, v. 22, n. 2, p. 291299, 1998.

SOUZA, Z. M.; ALVES, M. C. Propriedades químicas de um Latossolo Vermelho distrófico de Cerrado sob diferentes usos e manejos. Revista Brasileira de Ciência do Solo, Viçosa, v. 27, n. 1, p. 133-139, 2003.

UNIÃO DA INDÚSTRIA DE CANA-DE-AÇÚCAR (Unica). Previsão de safra 2008/2009. Informativo da Unica, São Paulo, n. 76, p. 1-21, maio/jun. 2007.

VIEIRA JÚNIOR, P. A. et al. Produção brasileira de canade-açúcar e deslocamento da fronteira agrícola no Estado do Mato Grosso. Informações Econômicas, São Paulo, v. 38, n. 4, p. 58-77, 2008. 